Article

\title{
Estimation of Reliability in a Multicomponent Stress-Strength System for the Exponentiated Moment-Based Exponential Distribution
}

\author{
G. Srinivasa Rao ${ }^{1}\left(\mathbb{D}\right.$, Fiaz Ahmad Bhatti ${ }^{2}$, Muhammad Aslam ${ }^{3}(\mathbb{D})$ and Mohammed Albassam ${ }^{3, *(D)}$ \\ 1 Department of Statistics, School of Mathematical Sciences, CNMS, The University of Dodoma, P.O. Box 259, \\ Dodoma 41000, Tanzania; gaddesrao@gmail.com \\ 2 National College of Business Administration and Economics, Lahore 54000, Pakistan; \\ fiazahmad72@gmail.com \\ 3 Department of Statistics, Faculty of Science, King Abdulaziz University, Jeddah 21551, Saudi Arabia; \\ malbassam@kau.edu.sa \\ * Correspondence: aslam_ravian@hotmail.com or magmuhammad@kau.edu.sa
}

Received: 8 October 2019; Accepted: 17 November 2019; Published: 21 November 2019

check for updates

\begin{abstract}
A multicomponent system of k components with independent and identically distributed random strengths $X_{1}, X_{2}, \ldots X_{k}$, with each component undergoing random stress, is in working condition if and only if at least $\mathrm{s}$ out of $\mathrm{k}$ strengths exceed the subjected stress. Reliability is measured while strength and stress are obtained through a process following an exponentiated moment-based exponential distribution with different shape parameters. Reliability is gauged from the samples using maximum likelihood (ML) on the computed distributions of strength and stress. Asymptotic estimates of reliability are compared using Monte Carlo simulation. Application to forest data and to breaking strengths of jute fiber shows the usefulness of the model.
\end{abstract}

Keywords: exponentiated moment-based exponential distribution; reliability; stress; strength; maximum likelihood; Monte Carlo simulation

\section{Introduction}

Dara and Ahmad [1] introduced the moment-based exponential distribution. Hasnain et al. [2] introduced and studied properties of the exponentiated moment-based exponential distribution. The cumulative distribution function of the exponentiated moment-based exponential distribution is as follows:

$$
F_{X}(x ; \alpha, \beta)=\left(1-\left(1+\frac{x}{\beta}\right) e^{-\frac{x}{\beta}}\right)^{\alpha}, \text { for } x>0,
$$

and its probability density function is as follows:

$$
\mathrm{f}_{X}(x ; \alpha, \beta)=\frac{\alpha x e^{-\frac{x}{\beta}}}{\beta^{2}}\left(1-\left(1+\frac{x}{\beta}\right) e^{-\frac{x}{\beta}}\right)^{\alpha-1}, \text { for } x>0,
$$

where $\alpha$ is the shape parameter and $\beta$ the scale parameter.

The moment distributions have a wide range of applications in different fields of study. Krumbein and Pettijohn [3] and Gy [4] examined the relationship between particle diameter and its frequency using moment distributions. Preston [5] used canonical distributions to characterize the relationship of land birds in England and Wales, breeding birds in Finland, mammals in the East Indies, and amphibian and reptiles in the West Indies. Zelen [6] introduced the use of weighted distributions to represent length-biased sampling in the case of cell kinetics and the early detection of disease. In addition, 
Zelen [7]) used length-biased sampling in the context of the disease screening and scheduling of patients' examinations. Brown [8] introduced low-density traffic streams in the context of studying various aspects of the traffic streams. Warren [9] studied the role of statistical distribution in different cases related to forestry and forest products. Taillie et al. [10] used weighted distributions in the context of modelling populations of fish stocks.

Let $G$ be the cumulative distribution function of $Y$ and $F$ be the cumulative distribution function of $X_{1}, X_{2}, \ldots X_{k}$. Bhattacharyya and Johnson [11] developed multicomponent reliability for a stress-strength model as follows:

$$
\begin{aligned}
& R_{s, k}=P\left(\text { at least } s \text { variables among } X_{1}, X_{2}, \ldots X_{k} \text { exceed } Y\right) \\
&=\sum_{i=s}^{k}\left(\begin{array}{c}
k \\
i
\end{array}\right) \int_{-\infty}^{\infty}(1-G(y))^{i}(G(y))^{k-i} d F(y)
\end{aligned}
$$

where $X_{1}, X_{2}, \ldots, X_{k}$ are independent and identically distributed random variables with cumulative distribution function $F$. This system has a common random stress $Y$. The probability, $R_{s, k}$, in Equation (3) is called reliability in a multicomponent stress-strength model. Enis and Geisser [12] estimated the probability of $Y<X$. Downtown [13] extended their results to the normal distribution; Awad and Gharraf [14] to the Burr distribution; McCool [15] to the Weibull; Nandi and Aich [16] to some distributions useful in lifetesting (a process done under controlled conditions to determine how and when a product will fail); Surles and Padgett [17] developed inference for reliability and stress-strength for a scaled Burr type $X$ distribution;and Raqab and Kundu [18] compared estimators of $P(Y<X)$ for a Burr type $X$ distribution. Their work was extended by Kundu and Gupta [19] for the exponential distribution, by Kundu and Gupta [20] for the Weibull distribution, by Raqab et al. [21] for the three-parameter exponential distribution, and by Kundu and Raqab [22] for the three-parameter Weibull distribution. Bhattacharyya and Johnson [11] expressed reliability in a multicomponent stress-strength model, as did Pandey and Uddin [23] with the Burr distribution. Rao and Kantam [24] estimated reliability in the multicomponent stress-strength model for a log-logistic distribution, Rao [25] for the exponential distribution, Rao et al. [26] for an inverse Rayleigh distribution, and Rao et al. [27] for the Burr XII distribution.

Consider a system with $k$ similar components and at least $s(1 \leq s \leq k)$ components operating simultaneously. It undergoes stress $Y$, which is a random variable with distribution function $G$. $F$ is the cumulative distribution function of minimal stresses causing the failure of a component. Then, the reliability of the system is the function $R_{s, k}$ given in Equation (3), which is the probability that the entire system does not fail at a specified time.

By exploring the literature and to the best of our knowledge, there is no work on the estimation of reliability in a multicomponent stress-strength system for the exponentiated moment-based exponential distribution. The objective of the paper was to estimate reliability in the multicomponent stress-strength model with respect to the exponentiated moment-based exponential distribution. We expected that the proposed method would be more efficient than the existing method.

\section{Estimation of $R_{s, k}$ by Maximum Likelihood}

The random variables $X$ and $Y$ are independently distributed. Each follows an exponentiated moment-based exponential distribution of parameters $\left(\alpha_{1}, \beta\right)$ for $X$ and $\left(\alpha_{2}, \beta\right)$ for $Y$, where $\beta$ is the unknown common scale parameter and the shape parameters $\alpha_{1}$ and $\alpha_{2}$ are unknown. 
The multicomponent stress-strength reliability for the exponentiated moment-based exponential distribution using Equation (3) is as follows:

$$
\begin{aligned}
& R_{s, k}= \sum_{i=s}^{k}\left(\begin{array}{c}
k \\
i
\end{array}\right) \int_{0}^{1}\left(1-t^{\alpha_{1}}\right)^{i}\left(t^{\alpha_{1}}\right)^{k-i} \alpha_{2} t^{\alpha_{2}-1} d t, \quad \text { where } t=1-\left(1+\frac{y}{\beta}\right) e^{-\frac{y}{\beta}} \\
&= \sum_{i=s}^{k}\left(\begin{array}{c}
k \\
i
\end{array}\right) \int_{0}^{1}(1-z)^{i}(z)^{k-i} \alpha_{2}\left(z^{\frac{1}{\alpha_{1}}}\right)^{\alpha_{2}-1} \frac{z^{\frac{1}{\alpha_{1}}-1}}{\alpha_{1}} d z, \quad \text { where } z=t^{\alpha_{1}} \\
&= v \sum_{i=s}^{k}\left(\begin{array}{c}
k \\
i
\end{array}\right) \int_{0}^{1}(1-z)^{i}(z)^{k-i+v-1} d z, \quad \text { where } v=\frac{\alpha_{2}}{\alpha_{1}} \\
&=v \sum_{i=s}^{k}\left(\begin{array}{c}
k \\
i
\end{array}\right) \mathrm{B}(i+1, k-i+v), \quad \text { where } \mathrm{B}(\mathrm{m}, \mathrm{n}) \text { is the beta first kind function. }
\end{aligned}
$$

After simplification, we obtain the following:

$$
R_{s, k}=v \sum_{i=s}^{k} \frac{k !}{(k-i) !}\left(\prod_{j=0}^{i}(k+v-j)\right)^{-1}
$$

since $k$ and $i$ are integers and $v=\frac{\alpha_{2}}{\alpha_{1}}$ can be obtained in Equations (11) and (12).

Equation (5) is a multicomponent stress-strength reliability of the system.

Let $\left(X_{1}, X_{2}, \ldots, X_{n}\right)$ be a random sample of strength variables and $\left(Y_{1}, Y_{2}, \ldots, Y_{m}\right)$ be a random sample of stress variables following an exponentiated moment-based exponential distribution with shape parameters $\alpha_{1}$ and $\alpha_{2}$ and common scale parameter $\beta$. The log-likelihood associated with the observed sample is as follows:

$$
\begin{gathered}
L\left(\alpha_{1}, \alpha_{2}, \beta\right)=\prod_{i=1}^{n} f\left(x_{i}, \alpha_{1}, \alpha_{2}\right) \prod_{j=1}^{m} f\left(y_{j}, \alpha_{1}, \alpha_{2}\right), \\
\ln L=n \ln \alpha_{1}+m \ln \alpha_{2}-2(m+n) \ln \beta+\sum_{i=1}^{n} \ln x_{i}+\sum_{j=1}^{m} \ln y_{j}-\frac{1}{\beta} \sum_{i=1}^{n} x_{i}-\frac{1}{\beta} \sum_{j=1}^{m} y_{j} \\
+\left(\alpha_{1}-1\right) \sum_{i=1}^{n} \ln \left(1-\left(1+\frac{x_{i}}{\beta}\right) e^{-\frac{x_{i}}{\beta}}\right)+\left(\alpha_{2}-1\right) \sum_{j=1}^{m} \ln \left(1-\left(1+\frac{y_{i}}{\beta}\right) e^{-\frac{y_{i}}{\beta}}\right) .
\end{gathered}
$$

The maximum likelihood estimators $\hat{\alpha}_{1}, \hat{\alpha}_{2}$, and $\hat{\beta}$ are obtained iteratively as a solution of the following:

$$
\begin{gathered}
\frac{\partial \operatorname{lnL}}{\partial \alpha_{1}}=0 \Rightarrow \frac{n}{\alpha_{1}}+\sum_{i=1}^{n} \ln \left(1-\left(1+\frac{x_{i}}{\beta}\right) e^{-\frac{x_{i}}{\beta}}\right)=0 \Rightarrow \hat{\alpha}_{1}=\frac{-n}{\sum_{i=1}^{n} \ln \left(1-\left(1+\frac{x_{i}}{\beta}\right) e^{-\frac{x_{i}}{\beta}}\right)} \\
\frac{\partial \ln \mathrm{L}}{\partial \alpha_{2}}=0 \Rightarrow \frac{m}{\alpha_{2}}+\sum_{j=1}^{m} \ln \left(1-\left(1+\frac{y_{i}}{\beta}\right) e^{-\frac{y_{i}}{\beta}}\right)=0 \Rightarrow \hat{\alpha}_{2}=\frac{-m}{\sum_{j=1}^{m} \ln \left(1-\left(1+\frac{y_{i}}{\beta}\right) e^{-\frac{y_{i}}{\beta}}\right)^{\prime}} \\
\frac{\partial \ln \mathrm{L}}{\partial \beta}=0 \Rightarrow \frac{-2(n+m)}{\beta}+\frac{1}{\beta^{2}} \sum_{i=1}^{n} x_{i}+\frac{1}{\beta^{2}} \sum_{j=1}^{m} y_{j}-\left(\alpha_{1}-1\right) \sum_{i=1}^{n} \frac{x_{i}^{2} e^{-\frac{x_{i}}{\beta}}}{\left(1-\left(1+\frac{x_{i}}{\beta}\right) e^{-\frac{x_{i}}{\beta}}\right) \beta^{3}} \\
-\left(\alpha_{2}-1\right) \sum_{j=1}^{m} \frac{y_{j}^{2} e^{-\frac{y_{i}}{\beta}}}{\left(1-\left(1+\frac{y_{i}}{\beta}\right) e^{-\frac{y_{i}}{\beta}}\right) \beta^{3}}=0 .
\end{gathered}
$$


From Equations (8) and (9), we obtain the following:

$$
\begin{aligned}
& \hat{\alpha}_{1}=\frac{-n}{\sum_{i=1}^{n} \ln \left(1-\left(1+\frac{x_{i}}{\hat{\beta}}\right) e^{-\frac{x_{i}}{\hat{\beta}}}\right)^{\prime}} \\
& \hat{\alpha}_{2}=\frac{-m}{\sum_{j=1}^{m} \ln \left(1-\left(1+\frac{y_{i}}{\hat{\beta}}\right) e^{-\frac{y_{i}}{\hat{\beta}}},\right.},
\end{aligned}
$$

and $\hat{\beta}$ is the solution of the following non-linear equation:

$$
\begin{aligned}
& \begin{array}{l}
g(\beta)=0 \Leftrightarrow \\
\frac{-2(n+m)}{\beta}+\frac{1}{\beta^{2}} \sum_{i=1}^{n} \frac{x_{i}\left(1-e^{-\frac{x_{i}}{\beta}}\right)}{\left(1-\left(1+\frac{x_{i}}{\beta}\right) e^{-\frac{x_{i}}{\beta}}\right)}+\frac{n}{\sum_{k=1}^{n} \ln \left(1-\left(1+\frac{x_{i}}{\beta}\right) e^{-\frac{x_{i}}{\beta}}\right.} \sum_{i=1}^{n} \frac{x_{i}^{2} e^{-\frac{x_{i}}{\beta}}}{\left(1-\left(1+\frac{x_{i}}{\beta}\right) e^{-\frac{x_{i}}{\beta}}\right) \beta^{3}}+
\end{array} \\
& \frac{1}{\beta^{2}} \sum_{j=1}^{m} \frac{y_{j}\left(1-e^{-\frac{y_{i}}{\beta}}\right)}{\left(1-\left(1+\frac{y_{i}}{\beta}\right) e^{-\frac{y_{i}}{\beta}}\right)}+\frac{m}{\sum_{k=1}^{m} \ln \left(1-\left(1+\frac{y_{i}}{\beta}\right) e^{-\frac{y_{i}}{\beta}}\right.} \sum_{i=1}^{n} \frac{y_{j}^{2} e^{-\frac{y_{i}}{\beta}}}{\left(1-\left(1+\frac{y_{i}}{\beta}\right) e^{-\frac{y_{i}}{\beta}}\right) \beta^{3}}=0 \text {, }
\end{aligned}
$$

where $\hat{\beta}$ is a simple iterative solution of the non-linear equation $g(\beta)=0$. With the obtrusion $\hat{\beta}, \hat{\alpha}_{1}$ is obtained from Equation (10) and $\hat{\alpha}_{2}$ from Equation (11), and the maximum likelihood estimate of $R_{s, k}$ is as follows:

$$
\hat{R}_{s, k}=\hat{v} \sum_{i=s}^{k} \frac{k !}{(k-i) !}\left(\prod_{j=0}^{i}(k+\hat{v}-j)\right)^{-1}, \text { where } \hat{v}=\frac{\hat{\alpha}_{2}}{\hat{\alpha}_{1}} .
$$

The variances of the maximum likelihood estimators are the following:

$$
V\left(\hat{\alpha}_{1}\right)=\left(E\left(-\frac{\partial^{2} \ln L}{\partial \alpha_{1}^{2}}\right)\right)^{-1}=\frac{\alpha_{1}^{2}}{n} \text { and } V\left(\hat{\alpha}_{2}\right)=\left(E\left(-\frac{\partial^{2} \ln L}{\partial \alpha_{2}^{2}}\right)\right)^{-1}=\frac{\alpha_{2}^{2}}{m} .
$$

The asymptotic variance of an estimate of $R_{s, k}$, which is a function of two independent statistics $\alpha_{1}$ and $\alpha_{2}$, is given by Rao [28]:

$$
V\left(\hat{\mathrm{R}}_{s, k}\right) \cong V\left(\hat{\alpha}_{1}\right)\left(\frac{\partial R_{s, k}}{\partial \alpha_{1}}\right)^{2}+V\left(\hat{\alpha}_{2}\right)\left(\frac{\partial R_{s, k}}{\partial \alpha_{2}}\right)^{2} .
$$

Equation (16) yields the asymptotic variance of $\hat{R}_{s, k}$ :

$$
\frac{\partial \hat{R}_{1,3}}{\partial \alpha_{1}}=\frac{3 \hat{v}}{\hat{\alpha}_{1}(3+\hat{v})^{2}} \text { and } \frac{\partial \hat{R}_{1,3}}{\partial \alpha_{2}}=\frac{-3}{\hat{\alpha}_{1}(3+\hat{v})^{2}} .
$$

The derivatives of $R_{s, k}$ for $(s, k)=(1,3)$ and $(s, k)=(2,4)$ are obtained separately:

$$
\frac{\partial \hat{R}_{2,4}}{\partial \alpha_{1}}=\frac{12(7+2 \hat{v})}{\hat{\alpha}_{1}((3+\hat{v})(4+\hat{v}))^{2}} \text { and } \frac{\partial \hat{R}_{2,4}}{\partial \alpha_{2}}=\frac{-12(7+2 \hat{v})}{\hat{\alpha}_{1}((3+\hat{v})(4+\hat{v}))^{2}} .
$$

Thus,

$$
\begin{gathered}
V\left(\hat{R}_{1,3}\right)=\frac{9 \hat{v}^{2}}{(3+\hat{v})^{4}}\left(\frac{1}{n}+\frac{1}{m}\right), \\
V\left(\hat{R}_{2,4}\right)=\frac{144 \hat{v}^{2}(2 \hat{v}+7)^{2}}{((3+\hat{v})(4+\hat{v}))^{4}}\left(\frac{1}{n}+\frac{1}{m}\right) .
\end{gathered}
$$


Under the usual regularity conditions (Rao, [28]):

as $n \rightarrow \infty, m \rightarrow \infty, \frac{\hat{R}_{s, k}-R_{s, k}}{V\left(\hat{R}_{s, k}\right)} \stackrel{d}{\rightarrow} N(0,1)$.

The asymptotic $100(1-\alpha) \%$ confidence interval for $R_{s, k}$ is as follows:

$$
\left[\hat{R}_{s, k} \mp Z_{(1-\alpha / 2)}\left(V\left(\hat{R}_{s, k}\right)\right)^{1 / 2}\right] .
$$

The asymptotic $100(1-\alpha) \%$ confidence interval for $R_{1,3}$ is as follows:

$$
\left[\hat{R}_{1,3} \mp Z_{(1-\alpha / 2)} \frac{3 \hat{v}}{(3+\hat{v})^{2}}\left(\frac{1}{n}+\frac{1}{m}\right)^{1 / 2}\right],
$$

where $\hat{v}=\hat{\alpha}_{2} / \hat{\alpha}_{1}$.

The asymptotic $100(1-\alpha) \%$ confidence interval for $R_{2,4}$ is as follows:

$$
\left[\hat{R}_{2,4} \mp Z_{(1-\alpha / 2)} \frac{12 \hat{v}(2 \hat{v}+7)}{((3+\hat{v})(4+\hat{v}))^{2}}\left(\frac{1}{n}+\frac{1}{m}\right)^{1 / 2}\right],
$$

where $\hat{v}=\hat{\alpha}_{2} / \hat{\alpha}_{1}$ and $Z_{(1-\alpha / 2)}$ is the $(1-\alpha / 2)^{\text {th }}$ percentile of the standard normal distribution.

The following Algorithm 1 is used for the estimation of $R_{s, k}$

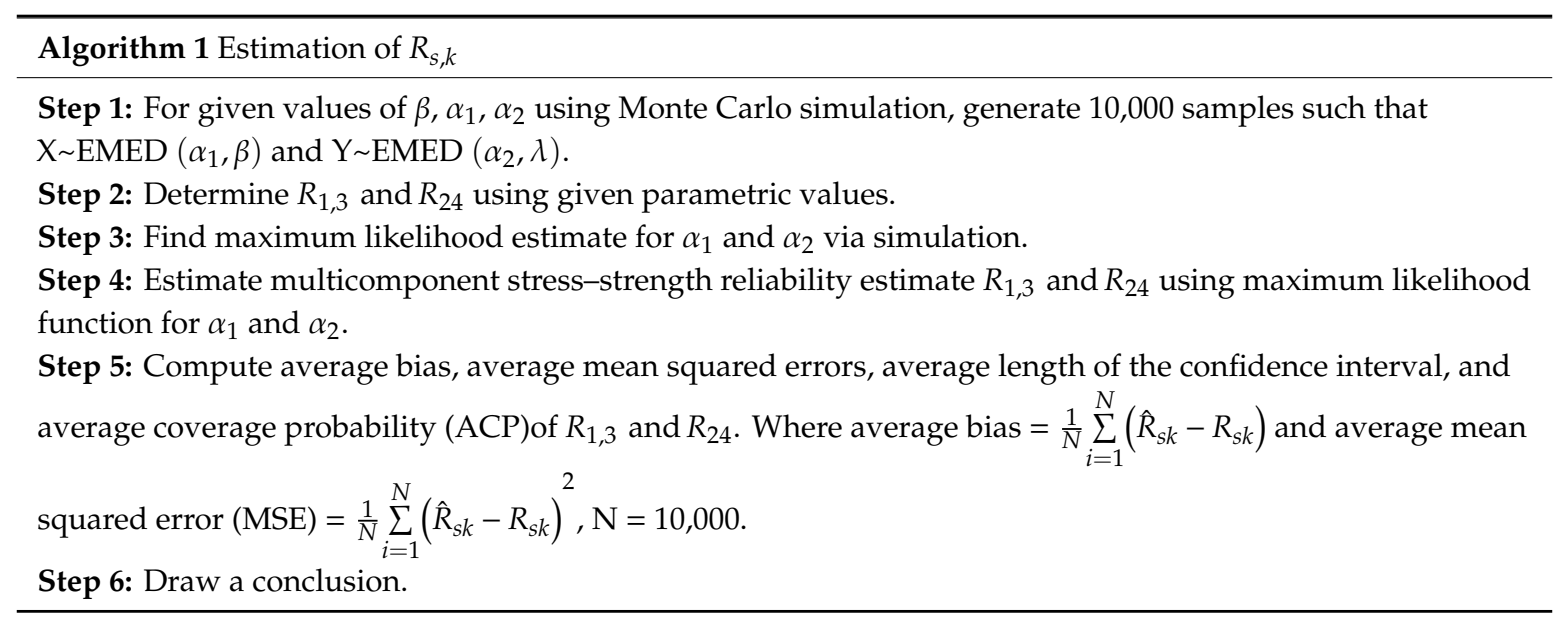

\section{Monte Carlo Simulation}

In this section, we carry out an extensive Monte Carlo simulation study to compare the performances of the maximum likelihood (ML) estimates of $R_{s, k}$. The comparisons are done based on point and interval estimations. The simulation study is carried out on 5000 simulated samples from exponentiated moment-based exponential distributed stress and strength variables for different sample sizes $n=m=15,20,25,30,35,40,45$, and 50 and for the combinations of the following parameters:

$$
\left(\alpha_{1}, \alpha_{2}\right)=(2.25,1.5),(2,1.5),(1.75,1.5),(1.5,1.5),(1.5,1.75),(1.5,2),(1.5,2.25) .
$$

We follow Bhattacharyya and Johnson [11] for the simulation procedure. We insert the estimates of $\beta, \alpha_{1}, \alpha_{2}$ into the expression of $v$ in Equation (5) to obtain the multicomponent value of reliability for $(s, k)=(1,3)$ and $(s, k)=(2,4)$. Tables 1 and 2 present the bias and the mean squared error for the estimates of reliability. Tables 3 and 4 present the simulated $95 \%$ confidence bounds and coverage probability of $R_{s, k}$. In the multicomponent stress-strength model, the true value of reliability using Equation (5) with the combination of $\left(\alpha_{1}, \alpha_{2}\right)$ in Equation (24) for $(s, k)=(1,3)$ is $0.8182,0.8000,0.7778$, $0.7500,0.7200,0.6923$, and 0.6667 . For $(s, k)=(2,4)$, the true value $y$ is successively $0.7013,0.6737$, 
$0.6405,0.6000,0.5574,0.5192$, and 0.4848 . For a fixed $\alpha_{1}$, the true value of reliability increases as $\alpha_{2}$ increases. For a fixed $\alpha_{2}$ and $(s, k)=(1,3)$ or $(s, k)=(2,4)$, reliability decreases as $\alpha_{1}$ increases. The true value of reliability decreases as $v$ increases.

Table 1. Bias of the simulated estimates of reliability $R_{s, k}$.

\begin{tabular}{ccccccccc}
\hline $\begin{array}{c}\text { Components } \\
(\boldsymbol{s}, \boldsymbol{k})\end{array}$ & $\begin{array}{c}\text { Sample Size } \\
(\boldsymbol{n}, \boldsymbol{m})\end{array}$ & \multicolumn{7}{c}{$\begin{array}{c}\text { Parameters } \\
\left(\boldsymbol{\alpha}_{\mathbf{1}}, \boldsymbol{\alpha}_{\mathbf{2}}\right)\end{array}$} \\
\hline & & $(2.25,1.5)$ & $(2.0,1.5)$ & $(1.75,1.5)$ & $(1.5,1.5)$ & $(1.5,1.75)$ & $(1.5,2.0)$ & $(1.5,2.25)$ \\
\hline & $(15,15)$ & 0.0223 & 0.0148 & 0.0069 & -0.0072 & -0.0218 & -0.0354 & -0.0510 \\
& $(20,20)$ & 0.0217 & 0.0147 & 0.0066 & -0.0057 & -0.0199 & -0.0335 & -0.0475 \\
& $(25,25)$ & 0.0216 & 0.0147 & 0.0062 & -0.0051 & -0.0188 & -0.0308 & -0.0446 \\
$(1,3)$ & $(30,30)$ & 0.0215 & 0.0146 & 0.0057 & -0.0032 & -0.0172 & -0.0291 & -0.0436 \\
& $(35,35)$ & 0.0215 & 0.0133 & 0.0052 & -0.0037 & -0.0152 & -0.0291 & -0.0419 \\
& $(40,40)$ & 0.0214 & 0.0132 & 0.0049 & -0.0028 & -0.0152 & -0.0273 & -0.0412 \\
& $(45,45)$ & 0.0212 & 0.0125 & 0.0043 & -0.0019 & -0.0151 & -0.0272 & -0.0409 \\
& $(50,50)$ & 0.0211 & 0.0123 & 0.0041 & -0.0016 & -0.0144 & -0.0264 & -0.0396 \\
& $(15,15)$ & 0.0344 & 0.0240 & 0.0125 & -0.0062 & -0.0251 & -0.0409 & -0.0581 \\
& $(20,20)$ & 0.0341 & 0.0236 & 0.0121 & -0.0052 & -0.0237 & -0.0401 & -0.0559 \\
& $(25,25)$ & 0.0339 & 0.0233 & 0.0118 & -0.0051 & -0.0231 & -0.0376 & -0.0533 \\
$(2,4)$ & $(30,30)$ & 0.0338 & 0.0231 & 0.0115 & -0.0027 & -0.0214 & -0.0360 & -0.0528 \\
& $(35,35)$ & 0.0337 & 0.0227 & 0.0111 & -0.0037 & -0.0192 & -0.0364 & -0.0512 \\
& $(40,40)$ & 0.0336 & 0.0225 & 0.0107 & -0.0027 & -0.0194 & -0.0344 & -0.0508 \\
& $(45,45)$ & 0.0332 & 0.0223 & 0.0102 & -0.0016 & -0.0194 & -0.0349 & -0.0507 \\
& $(50,50)$ & 0.0331 & 0.0218 & 0.0101 & -0.0013 & -0.0187 & -0.0337 & -0.0493 \\
\hline
\end{tabular}

Table 2. Mean squared error (MSE) of the simulated estimates of reliability $R_{s, k}$.

\begin{tabular}{ccccccccc}
\hline $\begin{array}{c}\text { Components } \\
(\boldsymbol{s}, \boldsymbol{k})\end{array}$ & $\begin{array}{c}\text { Sample Size } \\
(\boldsymbol{n}, \boldsymbol{m})\end{array}$ & \multicolumn{7}{c}{$\begin{array}{c}\text { Parameters } \\
\left(\boldsymbol{\alpha}_{1}, \boldsymbol{\alpha}_{\mathbf{2}}\right)\end{array}$} \\
\hline & & $(2.25,1.5)$ & $(2.0,1.5)$ & $(1.75,1.5)$ & $(1.5,1.5)$ & $(1.5,1.75)$ & $(1.5,2.0)$ & $(1.5,2.25)$ \\
\hline & $(15,15)$ & 0.0036 & 0.0041 & 0.0048 & 0.0059 & 0.0076 & 0.0097 & 0.0122 \\
& $(20,20)$ & 0.0027 & 0.0030 & 0.0034 & 0.0042 & 0.0057 & 0.0073 & 0.0092 \\
& $(25,25)$ & 0.0022 & 0.0024 & 0.0027 & 0.0033 & 0.0045 & 0.0058 & 0.0075 \\
$(1,3)$ & $(30,30)$ & 0.0019 & 0.0021 & 0.0022 & 0.0027 & 0.0036 & 0.0048 & 0.0064 \\
& $(35,35)$ & 0.0017 & 0.0018 & 0.0019 & 0.0023 & 0.0030 & 0.0043 & 0.0056 \\
& $(40,40)$ & 0.0015 & 0.0016 & 0.0016 & 0.0019 & 0.0027 & 0.0036 & 0.0050 \\
& $(45,45)$ & 0.0014 & 0.0014 & 0.0015 & 0.0017 & 0.0024 & 0.0034 & 0.0046 \\
& $(50,50)$ & 0.0013 & 0.0013 & 0.0013 & 0.0015 & 0.0021 & 0.0030 & 0.0041 \\
& $(15,15)$ & 0.0085 & 0.0092 & 0.0101 & 0.0114 & 0.0132 & 0.0153 & 0.0173 \\
& $(20,20)$ & 0.0065 & 0.0069 & 0.0074 & 0.0083 & 0.0101 & 0.0118 & 0.0134 \\
& $(25,25)$ & 0.0053 & 0.0056 & 0.0059 & 0.0066 & 0.0081 & 0.0096 & 0.0112 \\
$(2,4)$ & $(30,30)$ & 0.0047 & 0.0048 & 0.0048 & 0.0054 & 0.0066 & 0.0079 & 0.0097 \\
& $(35,35)$ & 0.0041 & 0.0041 & 0.0042 & 0.0047 & 0.0055 & 0.0071 & 0.0085 \\
& $(40,40)$ & 0.0037 & 0.0036 & 0.0036 & 0.0039 & 0.0050 & 0.0061 & 0.0077 \\
& $(45,45)$ & 0.0034 & 0.0033 & 0.0033 & 0.0036 & 0.0044 & 0.0058 & 0.0071 \\
& $(50,50)$ & 0.0032 & 0.0029 & 0.0030 & 0.0031 & 0.0039 & 0.0050 & 0.0065 \\
\hline
\end{tabular}


Table 3. Length of the $95 \%$ confidence interval of simulated reliability $R_{s, k}$.

\begin{tabular}{ccccccccc}
\hline $\begin{array}{c}\text { Components } \\
(\boldsymbol{s}, \boldsymbol{k})\end{array}$ & $\begin{array}{c}\text { Sample Size } \\
(\boldsymbol{n}, \boldsymbol{m})\end{array}$ & \multicolumn{7}{c}{$\begin{array}{c}\text { Parameters } \\
\left(\boldsymbol{\alpha}_{\mathbf{1}}, \boldsymbol{\alpha}_{\mathbf{2}}\right)\end{array}$} \\
\hline & & $(2.25,1.5)$ & $(2.0,1.5)$ & $(1.75,1.5)$ & $(1.5,1.5)$ & $(1.5,1.75)$ & $(1.5,2.0)$ & $(1.5,2.25)$ \\
\hline & $(15,15)$ & 0.1893 & 0.2122 & 0.2367 & 0.2651 & 0.2915 & 0.3105 & 0.3250 \\
& $(20,20)$ & 0.1643 & 0.1839 & 0.2055 & 0.2307 & 0.2537 & 0.2710 & 0.2837 \\
& $(25,25)$ & 0.1476 & 0.1649 & 0.1838 & 0.2070 & 0.2277 & 0.2429 & 0.2545 \\
$(1,3)$ & $(30,30)$ & 0.1347 & 0.1509 & 0.1684 & 0.1887 & 0.2080 & 0.2221 & 0.2331 \\
& $(35,35)$ & 0.1250 & 0.1395 & 0.1564 & 0.1752 & 0.1924 & 0.2061 & 0.2161 \\
& $(40,40)$ & 0.1170 & 0.1309 & 0.1462 & 0.1639 & 0.1802 & 0.1927 & 0.2025 \\
& $(45,45)$ & 0.1103 & 0.1234 & 0.1378 & 0.1543 & 0.1701 & 0.1820 & 0.1911 \\
& $(50,50)$ & 0.1048 & 0.1173 & 0.1308 & 0.1464 & 0.1614 & 0.1726 & 0.1813 \\
& $(15,15)$ & 0.2907 & 0.3186 & 0.3459 & 0.3732 & 0.3924 & 0.3998 & 0.3993 \\
& $(20,20)$ & 0.2535 & 0.2776 & 0.3020 & 0.3266 & 0.3435 & 0.3510 & 0.3513 \\
$(2,4)$ & $(25,25)$ & 0.2281 & 0.2495 & 0.2711 & 0.2939 & 0.3094 & 0.3162 & 0.3168 \\
& $(30,30)$ & 0.2086 & 0.2287 & 0.2487 & 0.2688 & 0.2837 & 0.2902 & 0.2910 \\
& $(35,35)$ & 0.1938 & 0.2118 & 0.2312 & 0.2498 & 0.2632 & 0.2696 & 0.2705 \\
& $(40,40)$ & 0.1814 & 0.1988 & 0.2165 & 0.2341 & 0.2467 & 0.2529 & 0.2539 \\
& $(45,45)$ & 0.1712 & 0.1875 & 0.2041 & 0.2207 & 0.2331 & 0.2388 & 0.2399 \\
& $(50,50)$ & 0.1627 & 0.1783 & 0.1939 & 0.2096 & 0.2214 & 0.2270 & 0.2281 \\
\hline
\end{tabular}

Table 4. Coverage probability of the simulated $95 \%$ confidence interval of reliability $R_{s, k}$.

\begin{tabular}{ccccccccc}
\hline $\begin{array}{c}\text { Components } \\
(\boldsymbol{s}, \boldsymbol{k})\end{array}$ & $\begin{array}{c}\text { Sample Size } \\
(\boldsymbol{n}, \boldsymbol{m})\end{array}$ & \multicolumn{7}{c}{$\begin{array}{c}\text { Parameters } \\
\left(\boldsymbol{\alpha}_{\mathbf{1}}, \boldsymbol{\alpha}_{\mathbf{2}}\right)\end{array}$} \\
\hline & & $(2.25,1.5)$ & $(2.0,1.5)$ & $(1.75,1.5)$ & $(1.5,1.5)$ & $(1.5,1.75)$ & $(1.5,2.0)$ & $(1.5,2.25)$ \\
\hline & $(15,15)$ & 0.9375 & 0.9366 & 0.9377 & 0.9414 & 0.9389 & 0.9316 & 0.9264 \\
& $(20,20)$ & 0.9372 & 0.9385 & 0.9436 & 0.9481 & 0.9398 & 0.9349 & 0.9285 \\
& $(25,25)$ & 0.9421 & 0.9418 & 0.9464 & 0.9455 & 0.9374 & 0.9365 & 0.9297 \\
$(1,3)$ & $(30,30)$ & 0.9381 & 0.9414 & 0.9537 & 0.9532 & 0.9463 & 0.9421 & 0.9350 \\
& $(35,35)$ & 0.9469 & 0.9430 & 0.9473 & 0.9476 & 0.9499 & 0.9426 & 0.9347 \\
& $(40,40)$ & 0.9450 & 0.9473 & 0.9521 & 0.9552 & 0.9474 & 0.9423 & 0.9373 \\
& $(45,45)$ & 0.9444 & 0.9449 & 0.9501 & 0.9536 & 0.9502 & 0.9391 & 0.9448 \\
& $(50,50)$ & 0.9447 & 0.9488 & 0.9513 & 0.9530 & 0.9496 & 0.9477 & 0.9418 \\
& $(15,15)$ & 0.9324 & 0.9320 & 0.9348 & 0.9376 & 0.9382 & 0.9302 & 0.9248 \\
& $(20,20)$ & 0.9346 & 0.9406 & 0.9406 & 0.9455 & 0.9371 & 0.9351 & 0.9311 \\
$(2,4)$ & $(25,25)$ & 0.9413 & 0.9396 & 0.9459 & 0.9448 & 0.9374 & 0.9354 & 0.9286 \\
& $(30,30)$ & 0.9359 & 0.9397 & 0.9531 & 0.9530 & 0.9451 & 0.9410 & 0.9357 \\
& $(35,35)$ & 0.9460 & 0.9406 & 0.9473 & 0.9470 & 0.9481 & 0.9412 & 0.9353 \\
& $(40,40)$ & 0.9431 & 0.9460 & 0.9514 & 0.9553 & 0.9456 & 0.9420 & 0.9383 \\
& $(45,45)$ & 0.9434 & 0.9443 & 0.9493 & 0.9538 & 0.9504 & 0.9392 & 0.9423 \\
& $(50,50)$ & 0.9440 & 0.9485 & 0.9507 & 0.9530 & 0.9480 & 0.9466 & 0.9412 \\
\hline
\end{tabular}

Table 1 shows the decrease of the biases for both cases $(s, k)=(1,3)$ and $(s, k)=(2,4)$, when both samplesizes $n$ and $m$ increase. Table 2 shows the decrease of the mean squared error with the increase of both sample sizes $n$ and $m$ for $(s, k)=(1,3)$ and $(s, k)=(2,4)$. Tables 1 and 2 then show the consistency of the maximum likelihood estimator of $R_{s, k}$. Reliability is biased negatively when $\alpha_{1} \leq \alpha_{2}$. Table 2 shows that the mean squared error decreases as $\alpha_{1}$ increases for a fixed value of $\alpha_{2}$ in both cases of $(s, k)$, and the mean squared error decreases as $\alpha_{2}$ increases for a fixed $\alpha_{1}$ in both cases of $(s, k)$.

Table 3 shows that the length of the confidence interval decreases when the sample size increases. Table 4 shows that the coverage probability is close to 0.95 , which we expected. The confidence intervals perform satisfactorily for all combinations of parameters.

\section{Application}

\subsection{Example with Forest Data}

The population study of the interrelated patterns, processes, flora, fauna, and ecosystems in forests is forest ecology. Logically, trees are an important component of forest research, but the wide variety of 
other life forms and abiotic components in most forests means that other elements, such as wildlife or soilnutrients, are often the focal point. Thus, forest ecology is a highly diverse and important branch of ecological study. A forest ecosystem is a natural woodland unit consisting of all plants, animals, and microorganisms in that area functioning together with all of the non-living physical factors of the environment. Characteristics and methodological approaches of forest ecology data most often followcertain distributions, namely canonical distributions in ecology.

We used the forest data and resource assessment of forestry in Vanuatu, 1990-2000, from the project of the University of Wellington, Victoria, Australia, the Department of Meteorology, and the Department of Forests. The data are available from Corrigan [29]. Forest loss is presented in Table 5. We fitted an exponentiated moment-based exponential distribution for each dataset. We used the Kolmogorov-Smirnov test as a goodness-of-fit test of the exponentiated moment-based exponential distribution. The Kolmogorov-Smirnov distance between the empirical and the fitted distributions was 0.23 with a $p$-value of 0.44 for the first dataset and 0.16 with a $p$-value of 0.83 for the second dataset. Therefore, the fit is satisfactory.

Table 5. Statistics of forest loss derived from satellite data analysis of 1990-2000.

\begin{tabular}{ccc}
\hline Vanuatu Island (Group) & Area (Hectare) & Loss of Forest (Hectare) \\
\hline Torres Islands & 11.52 & 45.8 \\
Banks Islands & 75.359 & 56.8 \\
Santo & 423.897 & 1114.4 \\
Maewo & 30.39 & 217 \\
Aoba & 40.566 & 210.4 \\
Pentecost & 49.49 & 249 \\
Malakula & 206.756 & 293.4 \\
Ambrym & 73.246 & 447.4 \\
Epi & 53.324 & 190.3 \\
Efate & 97.004 & 302.5 \\
Erromango & 88.874 & 666 \\
Tanna & 56.668 & 811.6 \\
Aneityum & 17.21 & 73 \\
\hline
\end{tabular}

We found $\hat{\alpha}_{1}=0.33$ and $\hat{\alpha}_{2}=0.89, \hat{R}_{1,3}=0.53$ and $\hat{R}_{2,4}=0.32$. The $95 \%$ confidence interval for $R_{1,3}$ was [0.3344, 0.7176] and for $R_{2,4}$ it was [0.1021, 0.5257].

\subsection{Example with Breaking Strength Data}

We used the empirical datasets of Xia et al. [30] about the breaking strengths of jute fiber at gauge lengths of 10 and $20 \mathrm{~mm}$. The diameters of jute fibers were measured with an XSP-8CA digital biological microscope (Shanghai Optical Instrument Factory, Shanghai, China). For the sake of simplicity, we considered the fibers to be perfectly cylindrical. We tested 20 fiber samples at four gauge lengths $(5,10$, 15 , and $20 \mathrm{~mm}$ ). The ultimate cells constrained by the environment break when increasing the tensile strength applied on the jute filament, until the length of the ultimate cell reaches a minimum load, which can no longer buildup to its breaking strength measured in megapascal (MPa), and is given as follows:

Dataset 1 (10 millimeters) MPa: 693.73, 704.66, 323.83, 778.17, 123.06, 637.66, 383.43, 151.48, 108.94, 50.16, 671.49, 183.16, 257.44, 727.23, 291.27, 101.15, 376.42, 163.40, 141.38, 700.74, 262.90, 353.24, 422.11, 43.93, 590.48, 212.13, 303.90, 506.60, 530.55, 177.25;

Dataset 2 (20 millimeters) MPa: 71.46, 419.02, 284.64, 585.57, 456.60, 113.85, 187.85, 688.16, 662.66, $45.58,578.62,756.70,594.29,166.49,99.72$, 707.36, 765.14, 187.13, 145.96, 350.70, 547.44, 116.99, 375.81, $581.60,119.86,48.01,200.16,36.75,244.53,83.55$.

We fitted an exponentiated moment-based exponential distribution for each dataset. We used the Kolmogorov-Smirnov test as a goodness-of-fit test of the exponentiated moment-based exponential 
distribution. The Kolmogorov-Smirnov distance between the empirical and the fitted distribution was 0.11 with a $p$-value $=0.89$ for the first dataset and 0.15 with a $p$-value $=0.47$ for the second dataset, indicating satisfactory fits.

We found $\hat{\alpha}_{1}=0.77$ and $\hat{\alpha}_{2}=0.76, \hat{R}_{1,3}=0.75$ and $\hat{R}_{2,4}=0.60$. The $95 \%$ confidence interval for $R_{1,3}$ was [0.6551,0.8449] and for $R_{2,4}$ it was [0.4634, 0.7366].

\section{Comparison with Existing Distribution}

We compared our results with the existing work of Rao et al. [27] at $\left(\alpha_{1}, \alpha_{2}\right)=(1.5,1.5)$ and the results are displayed in Table 6. They show that average MSEs and average confidence lengths (ACLs) are lesser than the existing results based on Burr XII distribution. The average coverage probability also shows better performance than Burr XII distribution. Thus, as compared with Burr XII distribution, our results based on exponentiated moment-based exponential distribution perform well with respect to the results of multicomponent stress-strength.

Table 6. Comparison of MSE, the average confidence length (ACL), and 95\% average coverage probability $(\mathrm{ACP})$ at $\left(\alpha_{1}, \alpha_{2}\right)=(1.5,1.5)$ for two distributions. EMED, exponentiated moment-based exponential distribution.

\begin{tabular}{cccccccc}
\hline \multirow{2}{*}{$(s, k)$} & $(n, m)$ & \multicolumn{3}{c}{ EMED } & \multicolumn{3}{c}{ Burr XII Distribution } \\
\cline { 3 - 8 } & & MSE & ACL & ACP & MSE & ACL & ACP \\
\hline$(1,3)$ & $(15,15)$ & 0.0059 & 0.2651 & 0.9264 & 0.0105 & 0.3722 & 0.9403 \\
& $(20,20)$ & 0.0042 & 0.2307 & 0.9331 & 0.0078 & 0.3239 & 0.9430 \\
& $(25,25)$ & 0.0033 & 0.2070 & 0.9305 & 0.0059 & 0.2922 & 0.9470 \\
& $(30,30)$ & 0.0027 & 0.1887 & 0.9382 & 0.0051 & 0.2684 & 0.9380 \\
$(2,4)$ & $(15,15)$ & 0.0114 & 0.3732 & 0.9226 & 0.0136 & 0.4247 & 0.9347 \\
& $(20,20)$ & 0.0083 & 0.3266 & 0.9305 & 0.0099 & 0.3730 & 0.9397 \\
& $(25,25)$ & 0.0066 & 0.2939 & 0.9298 & 0.0078 & 0.3367 & 0.9440 \\
& $(30,30)$ & 0.0054 & 0.2688 & 0.9380 & 0.0067 & 0.3086 & 0.9330 \\
\hline
\end{tabular}

\section{Conclusions}

We introduced the exponentiated moment-based exponential distribution in the study of multicomponent stress-strength reliability, when stress and strength variables concern the same population. We used Monte Carlo simulations to compute large-sample confidence intervals. The larger the sample size, the smaller the bias and the mean squared error. The average bias is negative when $\alpha_{1} \leq \alpha_{2}$; otherwise, the bias is positive for $(s, k)=(1,3)$ and $(s, k)=(2,4)$. The mean squared error decreases as $\alpha_{1}$ increases for a fixed $\alpha_{2}$ and increases as $\alpha_{2}$ increases for a fixed $\alpha_{1}$ for $(s, k)=(1,3)$ and $(s, k)=(2,4)$. The confidence intervals shrink with larger sample sizes and the performance of the coverage probability is satisfactory.

Author Contributions: Conceptualization, G.S.R., F.A.B., M.A. (Muhammad Aslam) and M.A. (Mohammed Albassam); Methodology, G.S.R., F.A.B., M.A. (Muhammad Aslam); Software, G.S.R.; Validation, M.A. (Muhammad Aslam); Formal analysis, G.S.R., F.A.B., M.A. (Muhammad Aslam); Investigation, G.S.R., F.A.B., M.A. (Muhammad Aslam); Writing—review and editing, G.S.R., F.A.B., M.A. (Muhammad Aslam).

Funding: This article was funded by the Deanship of Scientific Research (DSR) at King Abdulaziz University, Jeddah, Saudi Arabia. The authors, therefore, acknowledge with thanks DSR technical and financial support.

Acknowledgments: The authors are deeply thankful to the editor and reviewers for their valuable suggestions to improve the quality of this manuscript.

Conflicts of Interest: The authors declare no conflict of interest. 


\section{Glossary}

EMED-Exponentiated moment-based exponential distribution

$\alpha$-Shape parameter

$\beta$-Scale parameter

$R_{s, k}$-Multicomponent stress-strength reliability

$B(m, n)$-Beta first kind function

MSE-Mean squared error

$\mathrm{ACP}$-Average coverage probability

ACL-Average confidence length

ML-Maximum likelihood

\section{References}

1. Dara, S.T.; Ahmad, M. Recent Advances in Moment Distributions and their Hazard Rate. Ph.D. Thesis, National College of Business Administration and Economics, Lahore, Pakistan, 2012.

2. Hasnain, S.A.; Iqbal, Z.; Munir, A. On exponentiated moment exponential distribution. Pak. J. Stat. 2015, 31, 267-280.

3. Krumbein, W.C.; Pettijohn, F.J. Manual of Sedimentary Petrography: Preparation for Analysis, Mechanical Analysis and Statistical Analysis; Shape Analysis, Mineralogical Analysis, Chemical Analysis and Mass Properties; Appleton Century-Crofts, Inc.: New York, NY, USA, 1938; pp. 230-233.

4. Gy, P. Sampling of Particulate Material: Theory and Practice; Elsevier: Amsterdam, The Netherlands, 1982.

5. Preston, F.W. The canonical distribution of commonness and rarity. Ecology 1962, 43, 186-215. [CrossRef]

6. Zelen, M. Theory of Early Detection of Breast Cancer in the General Population. Breast Cancer 1976, 2 , 287-301.

7. Zelen, M. Problems in Cell Kinetics and the Early Detection of Disease, in Reliability and Biometry; Proschan, F., Serfling, R.J., Eds.; SIAM: Philadelphia, PA, USA, 1974; pp. 701-706.

8. Brown, M. Low Density Traffic Streams. Adv. Appl. Probab. 1972, 4, 177-192. [CrossRef]

9. Warren, W. Statistical Distributions in Forestry and Forest Products Research; Reidel: Dordrecht, The Netherlands, 1975; Volume 2, pp. 360-384.

10. Taillie, C.; Patil, G.P.; Hennemuth, R. Modelling and analysis of recruitment distributions. Ecol. Environ. Stat. 1995, 2, 315-329. [CrossRef]

11. Bhattacharyya, G.K.; Johnson, R.A. Estimation of reliability in multicomponent stress-strength model. J. Am. Stat. Assoc. 1974, 69, 966-970. [CrossRef]

12. Enis, P.; Geisser, S. Estimation of the probability that $Y<X$. J. Am. Stat. Assoc. 1971, 66, 162-168.

13. Downtown, F. The estimation of $P(Y<X)$ in the normal case. Technometrics 1973, 15, 551-558.

14. Awad, M.; Gharraf, K. Estimation of $P(Y<X)$ in Burr case: A comparative study. Commun. Stat.-Simul. Comput. 1986, 15, 389-403.

15. McCool, J.I. Inference on $P(Y<X)$ in the Weibull case. Commun. Stat.-Simul. Comput. 1991, 20, 129-148.

16. Nandi, S.B.; Aich, A.B. A note on estimation of $P(Y>X)$ for some distributions useful in life-testing. IAPQR Trans. 1994, 19, 35-44.

17. Surles, J.G.; Padgett, W.J. Inference for reliability and stress-strength for a scaled Burr Type X distribution. Lifetime Data Anal. 2001, 7, 187-200. [CrossRef] [PubMed]

18. Raqab, M.Z.; Kundu, D. Comparison of different estimators of $P(Y<X)$ for a scaled Burr Type $X$ distribution. Commun. Stat.-Simul. Comput. 2005, 34, 465-483.

19. Kundu, D.; Gupta, R.D. Estimation of $P(Y<X)$ for the generalized exponential distribution. Metrika 2005, 61, 291-308.

20. Kundu, D.; Gupta, R.D. Estimation of $P(Y<X)$ for Weibull distribution. IEEE Trans. Reliab. 2006, 55, $270-280$.

21. Raqab, M.Z.; Madi, M.T.; Kundu, D. Estimation of $P(Y<X)$ for the 3-parameter generalized exponential distribution. Commun. Stat.-Theory Methods 2008, 37, 2854-2864.

22. Kundu, D.; Raqab, M.Z. Estimation of $\mathrm{R}=P(Y<X)$ for three-parameter Weibull distribution. Stat. Probab. Lett. 2009, 79, 1839-1846.

23. Pandey, M.; Uddin, M.B. Estimation of reliability in multi-component stress-strength model following a Burr distribution. Microelectr. Reliab. 1991, 31, 21-25. [CrossRef] 
24. Rao, G.S.; Kantam, R.R.L. Estimation of reliability in multicomponent stress-strength model: Log-logistic distribution. Electron. J. Appl. Stat. Anal. 2010, 3, 75-84.

25. Rao, G.S. Estimation of reliability in multicomponent stress-strength based on generalized exponential distribution. Colomb. J. Stat. 2012, 35, 67-76.

26. Rao, G.S.; Aslam, M.; Kundu, D. Burr-XII distribution parametric estimation and estimation of reliability of multicomponent stress-strength. Commun. Stat.-Theory Methods 2015, 44, 4953-4961. [CrossRef]

27. Rao, G.S.; Kantam, R.R.L.; Rosaiah, K.; Reddy, J.P. Estimation of reliability in multicomponent stress-strength based on inverse Rayleigh distribution. J. Stat. Appl. Probab. 2013, 2, 261-267. [CrossRef]

28. Rao, C.R. Linear Statistical Inference and Its Applications; Wiley Eastern Limited: New Delhi, India, 1973.

29. Corrigan, H. Forest Data and Resource Assessment of Forestry in Vanuatu; MAQFF: Port-Vila, Vanuatu, 2009.

30. Xia, Z.P.; Yu, J.Y.; Cheng, L.D.; Liu, L.F.; Wang, W.M. Study on the breaking strength of jute fibers using modified Weibull distribution. J. Compos. Part A 2009, 40, 54-59. [CrossRef]

(C) 2019 by the authors. Licensee MDPI, Basel, Switzerland. This article is an open access article distributed under the terms and conditions of the Creative Commons Attribution (CC BY) license (http://creativecommons.org/licenses/by/4.0/). 